\title{
Almost squares in arithmetic progression (II)
}

\author{
by
}

Anirban Mukhopadhyay (Allahabad) and T. N. Shorey (Mumbai)

1. Introduction. For an integer $\nu>1$, we denote by $P(\nu)$ and $\omega(\nu)$ the greatest prime factor of $\nu$ and the number of distinct prime divisors of $\nu$, respectively. Further we put $P(1)=1$ and $\omega(1)=0$. Let $n, d, k, b, y$ be positive integers such that $b$ is square free, $d>1, k \geq 3$ and $P(b) \leq k$. We consider the equation

$$
n(n+d) \ldots(n+(k-1) d)=b y^{2} \quad \text { in } n, d, k, b, y \text { with } P(b) \leq k .
$$

For an account of results on (1), we refer to [8] and [9]. Shorey and Tijdeman [10] proved that (1) with $\operatorname{gcd}(n, d)=1$ implies that $k$ is bounded by an effectively computable number depending only on $\omega(d)$. Further Shorey [8, p. 489] showed that the assumption $\operatorname{gcd}(n, d)=1$ can be relaxed to $d \nmid n$ in the preceding result. On the other hand, we observe that (1) may have infinitely many solutions in the case $d \mid n$. Next Saradha and Shorey [7] showed that (1) with $b=1$ and $k \geq 4$ is not possible whenever $\omega(d)=1$. It has also been shown in [7] that (1) with $P(b)<k, d \nmid n, \omega(d)=1$ and $k \geq 10$ does not hold. In this paper, we prove

Theorem 1. Let $4 \leq k \leq 9, P(b)<k$ and $\omega(d)=1$ such that $d \nmid n$. Then (1) does not hold unless $n=75, d=23, k=4, b=6, y=4620$.

The case $k=3$ remains open even when $b=1$. Next we consider (1) with $P(b)=k$. Saradha and Shorey [7] showed that (1) with $P(b)=k$, $\operatorname{gcd}(n, d)=1$ and $\omega(d)=1$ implies that $k \leq 29$. We prove

Theorem 2. Let $7 \leq k \leq 29$ and $P(b)=k$. Assume that $\omega(d)=1$ and $d \nmid n$. Then (1) does not hold.

As stated above, the assumption $d \nmid n$ is necessary in the above theorems. The case $k=5$ in Theorem 2 remains unresolved. The proofs of Theorems 1 and 2 depend on the theory of linear forms in logarithms. This is a new element in the proof. By combining the results stated above, we have

2000 Mathematics Subject Classification: Primary 11D61. 
TheOREM 3. Let $k \neq 3,5$. Then $(1)$ with $\omega(d)=1$ and $d \nmid n$ implies that $n=75, d=23, k=4, b=6, y=4620$.

Fermat (see [4, p. 21]) stated that there are no four squares in an arithmetic progression and Euler (see [3, p. 635]) proved that (1) with $\operatorname{gcd}(n, d)=1, k=4, b=1$ is not possible. Further Obláth [5] showed that (1) with $\operatorname{gcd}(n, d)=1, k=5, b=1$ does not hold. We obtain the following extension of the result of Obláth.

TheOrem 4. Equation (1) with $\operatorname{gcd}(n, d)=1, k=5, P(b)<k$ does not hold.

We compute using SIMATH the Mordell group of an elliptic curve for the proof of Theorem 4. By (1), we write

$$
n+i d=a_{i} x_{i}^{2}, \quad P\left(a_{i}\right) \leq k, a_{i} \text { square free for } 0 \leq i<k,
$$

where $x_{i}$ are positive integers. Further we put $R=\left\{a_{0}, a_{1}, \ldots, a_{k-1}\right\}$.

We thank Professor Frits Beukers for his remarks. We also thank the referee for his comments on an earlier draft of this paper.

2. Lemmas. We start with an estimate of Baker and Wüstholz [2] from the theory of linear forms in logarithms. The height of an algebraic number is defined as the maximum of the absolute values of the coefficients of its minimal polynomial with relatively prime integer coefficients. Let $\alpha_{1}, \ldots, \alpha_{n}$ be algebraic numbers different from 0,1 and let $\log \alpha_{1}, \ldots, \log \alpha_{n}$ be the principal logarithms. Let $K$ be the field generated by $\alpha_{1}, \ldots, \alpha_{n}$ over $\mathbb{Q}$ and $d$ be the degree of $K$ over $\mathbb{Q}$. Assume that the heights of $\alpha_{1}, \ldots, \alpha_{n}$ do not exceed $A_{1}, \ldots, A_{n}$, respectively, where $A_{i} \geq e$ for $1 \leq i \leq n$. Let $b_{1}, \ldots, b_{n}$ be rational integers of absolute values not exceeding $B$ where $B \geq e$. We put

$$
\Lambda=b_{1} \log \alpha_{1}+\ldots+b_{n} \log \alpha_{n}
$$

We have

LEMMA 1. If $\Lambda \neq 0$, then

$$
\log |\Lambda|>-(16 n d)^{2(n+2)} \log A_{1} \ldots \log A_{n} \log B \text {. }
$$

The next result is due to Baker and Davenport [1].

Lemma 2. Let $\theta, \beta, C$ be real numbers with $C>1$. Suppose $K>6$. For any positive integer $M$, let $p$ and $q$ be integers satisfying

$$
1 \leq q \leq K M, \quad|\theta q-p|<2(K M)^{-1} .
$$

Then, if

$$
\|q \beta\| \geq 3 K^{-1}
$$


there is no solution of

$$
|m \theta-n+\beta|<C^{-m}
$$

in the range

$$
\frac{\log K^{2} M}{\log C}<m<M .
$$

Now we apply Lemmas 1 and 2 to solve certain simultaneous Pell's equations as in Baker and Davenport [1].

Lemma 3. Let $\delta \in\{-1,1\}$. Consider the following set of simultaneous Pell's equations:

$$
\begin{aligned}
X^{2}+2 & =6 Y^{2}, & (X+\delta)^{2}+2 & =3 Z^{2} ; \\
X^{2}-6 & =3 Y^{2}, & (X+\delta)^{2}-2 & =2 Z^{2} ; \\
X^{2}-3 & =6 Y^{2}, & (X+2 \delta)^{2}+1 & =2 Z^{2} ; \\
X^{2}+2 & =3 Y^{2}, & (X+\delta)^{2}+2 & =2 Z^{2} ; \\
X^{2}+4 & =2 Y^{2}, & (X+2 \delta)^{2}+6 & =6 Z^{2} .
\end{aligned}
$$

These equations have no solutions in positive integers $X, Y, Z$ other than $X=2, Y=1, Z=1$ with $\delta=-1$ for (3), $X=9, Y=5, Z=7$ with $\delta=1$ and $X=3, Y=1, Z=1$ with $\delta=-1$ for (4), $X=3, Y=1, Z=1$ with $\delta=-1$ for (5), $X=1, Y=1, Z=1$ with $\delta=-1$ for (6), $X=2, Y=2$, $Z=1$ and $X=14, Y=10, Z=5$ with $\delta=-1$ for $(7)$.

Proof. We follow Baker and Davenport [1] for the proof. The computations required for the proof are carried out using MATHEMATICA. By factorising the above equations, it is enough to solve the following exponential equations in non-negative integers $m$ and $n$ :

$$
\begin{gathered}
(1+\sqrt{3})(2+\sqrt{3})^{m}+(1-\sqrt{3})(2-\sqrt{3})^{m}-(2+\sqrt{6})(5+2 \sqrt{6})^{n} \\
-(2-\sqrt{6})(5-2 \sqrt{6})^{n}=2 \delta \\
(2+\sqrt{2})(3+2 \sqrt{2})^{n}+(2-\sqrt{2})(3-2 \sqrt{2})^{n}-(3-\sqrt{3})(2+\sqrt{3})^{m} \\
-(3+\sqrt{3})(2-\sqrt{3})^{m}=2 \delta \\
(1+\sqrt{2})(3+2 \sqrt{2})^{m}+(1-\sqrt{2})(3-2 \sqrt{2})^{m}-(3+\sqrt{6})(5+2 \sqrt{6})^{n} \\
-(3-\sqrt{6})(5-2 \sqrt{6})^{n}=4 \delta \\
\sqrt{2}(3+2 \sqrt{2})^{n}-\sqrt{2}(3-2 \sqrt{2})^{n}-(1+\sqrt{3})(2+\sqrt{3})^{m} \\
-(1-\sqrt{3})(2-\sqrt{3})^{m}=2 \delta \\
(2+2 \sqrt{2})(3+2 \sqrt{2})^{m}+(2-2 \sqrt{2})(3-2 \sqrt{2})^{m}-\sqrt{6}(5+2 \sqrt{6})^{n} \\
+\sqrt{6}(5-2 \sqrt{6})^{n}=-4 \delta .
\end{gathered}
$$

We check that $m>n$. By Lemma 1 , we derive that $m<10^{26}$. Next we apply Lemma 2 with $M=10^{26}$ and $K=10^{13}$ to conclude that $m<90$. By 
a computer search we find that all the solutions are given in the statement of Lemma 3.

For the further proofs it may be convenient to mention some standard arguments which are used repeatedly, sometimes without further reference. A square cannot be congruent to 2 modulo 3 . If $d$ is odd, it cannot happen that $a_{i}=a_{i+2}=1$ since it would follow that $x_{i+2}^{2}-x_{i}^{2}=2 d$, whereas the difference of two squares can never be 2 modulo 4 . If $p$ divides $n$, we see from (2) that $\left(\frac{i d}{p}\right)=\left(\frac{a_{i}}{p}\right)$. In particular, if $p=7$ and $a_{i} \in\{1,2,3,6\}$, then 3 divides $a_{i}$ if and only if $\left(\frac{i d}{p}\right)=-1$. Also, if 3 divides $n+i d$, then neither $a_{i-1}=a_{i+1}$ nor $a_{i+1}=a_{i+2}$ is possible.

As stated in Section 1, we have the following result of Euler and we include the proof for the sake of completeness.

LEMMA 4. Equation (1) with $k=4, b=1$ is not possible.

Proof. The proof depends on the result that the equation

$$
x^{4}-x^{2} y^{2}+y^{4}=z^{2} \quad \text { in positive integers } x, y, z \text { with } \operatorname{gcd}(x, y)=1
$$

has no solution other than $x=y=z=1$ (see Mordell [4, p. 20]). Assume (1) with $k=4$ and $b=1$. There is no loss of generality in assuming that $\operatorname{gcd}(n, d)=1$. Then we see from $(2)$ that $a_{0}=3, a_{1}=2, a_{2}=1, a_{3}=6$ or $a_{0}=6, a_{1}=1, a_{2}=2, a_{3}=3$ or $a_{0}=a_{1}=a_{2}=a_{3}=1$. The last possibility is excluded since it implies (8) with $z>1$ (see Mordell [4, p. 21]). Next we exclude the first possibility and the proof for the second is similar. By using $3(n+d)=2 n+(n+3 d)$, we observe that $x_{1}^{2}=x_{0}^{2}+x_{3}^{2}$. Since $x_{0}$ is odd and $x_{3}$ is even, we derive that $x_{0}=r^{2}-s^{2}$ and $x_{3}=2 r s$ where $r>s>0$ are integers such that $\operatorname{gcd}(r, s)=1$ and $r \not \equiv s(\bmod 2)$. Then $d=2 x_{3}^{2}-x_{0}^{2}=10 r^{2} s^{2}-r^{4}-s^{4}$ and $x_{2}^{2}=3 x_{0}^{2}+2 d=r^{4}+s^{4}+14 r^{2} s^{2}$. Next we write $x=r+s, y=r-s$ and we observe that $x>y>0$ with $\operatorname{gcd}(x, y)=1$ since $\operatorname{gcd}(r, s)=1$ such that $r \not \equiv s(\bmod 2)$. Then we obtain (8) with $z=x_{2}>1$ and this is a contradiction.

There are infinitely many pairs $(n, d)$ of relatively prime integers satisfying (1) with $k=4$ (see Tijdeman [11]). On the other hand, we apply Lemma 3 to show that there is no pair $(n, d)$ of relatively prime integers other than $(75,23)$ satisfying $(1)$ with $k=4$ whenever $d$ is a power of an odd prime.

LemMA 5. Let $d$ be an odd prime power. Then (1) with $\operatorname{gcd}(n, d)=1$, $k=4$ implies that $n=75, d=23, b=6, y=4620$.

Proof. We observe that $R \subset\{1,2,3,6\}$. By Lemma 4, we derive that $|R| \neq 1$, 4. If $|R|=2$, we again use Lemma 4 to observe that exactly three $a_{i}$ 's are equal to 1 implying that $d$ is even. Thus $|R|=3$. Then at least one $a_{i}$ is divisible by 3 . Suppose that 3 divides $a_{0}$ and $a_{3}$. Then $\left(\frac{a_{1}}{3}\right)=\left(\frac{d}{3}\right)$ and 
$\left(\frac{a_{2}}{3}\right)=-\left(\frac{d}{3}\right)$. So either $a_{1}=1, a_{2}=2$ or $a_{1}=2, a_{2}=1$. Thus

$$
a_{0}=3, a_{1}=2, a_{2}=1, a_{3}=3 \text { or } a_{0}=3, a_{1}=1, a_{2}=2, a_{3}=3 .
$$

From the first possibility, we observe from (2) that

$$
n=3 x_{0}^{2}, \quad n+d=2 x_{1}^{2}, \quad n+2 d=x_{2}^{2}, \quad n+3 d=3 x_{3}^{2} .
$$

So $d=x_{3}^{2}-x_{0}^{2}$ implying $d=x_{3}+x_{0}$ and $x_{3}-x_{0}=1$ since $d$ is an odd prime power. Thus $d=2 x_{0}+1$ and we obtain from (9) the following equations:

$$
X^{2}+2=6 Y^{2}, \quad(X+1)^{2}+2=3 Z^{2}
$$

with $X=3 x_{0}+1, Y=x_{1}, Z=x_{2}$. This is (3) of Lemma 3 with $\delta=1$. Thus by Lemma 3 we conclude that this case is not possible. From the other possibility we get the following equations:

$$
X^{2}+2=6 Y^{2}, \quad(X-1)^{2}+2=3 Z^{2}
$$

with $X=3 x_{0}+2 \geq 5, Y=x_{2}, Z=x_{1}$. This is (3) of Lemma 3 with $\delta=-1$, which is not possible.

Thus we may suppose that 3 divides exactly one $a_{i}$. Let $3 \mid a_{0}$. We apply Legendre symbols as above to get the following two possibilities:

$$
a_{0}=3, a_{1}=2, a_{2}=1, a_{3}=1 \quad \text { or } a_{0}=3, a_{1}=2, a_{2}=1, a_{3}=2 .
$$

The first one gives the equations

$$
X^{2}-6=3 Y^{2}, \quad(X+1)^{2}-2=2 Z^{2}
$$

with $X=x_{2}-2, Y=x_{0}, Z=x_{1}$. Now we apply Lemma 3 with (4), $\delta=1$ to conclude that $x_{2}=11, x_{0}=5, x_{1}=7$. Thus $n=75, d=23, b=6$ and $y=4620$. The second possibility gives

$$
X^{2}-3=6 Y^{2}, \quad(X+2)^{2}+1=2 Z^{2}
$$

with $X=2 x_{1}-1, Y=x_{0}, Z=x_{2}$ contradicting Lemma 3.

We proceed as above to observe that $3 \mid a_{1}$ gives (6) with $\delta=1$ such that $X=x_{0}+1, Y=x_{1}, Z=x_{2}$ or $X=3 x_{0}+1, Y=3 x_{1}, Z=3 x_{2} ; 3 \mid a_{2}$ gives (6) with $\delta=-1$ such that $X=x_{0}+2, Y=x_{2}, Z=x_{1}$ or $X=3 x_{0}+2$, $Y=3 x_{2}, Z=3 x_{1} ; 3 \mid a_{3}$ gives (4) with $\delta=-1, X=x_{0}+3, Y=x_{3}, Z=x_{2}$ or (5) with $\delta=-1, X=2 x_{0}+3, Y=x_{3}, Z=x_{1}$. This is not possible by Lemma 3.

Lemma 6. Let $11 \leq k \leq 29$ be prime. Then (1) with $\operatorname{gcd}(n, d)=1$, $P(b)=k$ and $|R| \geq k-1$ does not hold.

Proof. Let $k=29$. Then $|R| \geq 28$. We observe that the primes 29, 23, $19,17,13,11,7$ divide at most $1,2,2,2,3,3,5$ distinct $a_{i}$ 's respectively. Thus there are at least 10 distinct $a_{i}$ 's composed only of primes 2, 3 and 5, a contradiction.

Thus $11 \leq k \leq 23$. If $k=17,19,23$ and $|R|=k$, we observe that the number of distinct $a_{i}$ 's composed only of $2,3,5$ is at least 9 . If $k=11,13$ 
and $|R|=k$, we see that the number of distinct $a_{i}$ 's composed of 2,3 is at least 5 . This is not possible. Therefore $|R|=k-1$.

Let $k=23$. There are exactly 8 distinct $a_{i}$ 's composed of 2,3 and 5 . Therefore the primes $23,19,17,13,11,7$ divide exactly $1,2,2,2,3,4$ distinct $a_{i}$ 's, respectively, such that none of these $a_{i}$ 's is divisible by more than one of the above primes. Now we observe that 11 divides $a_{0}, a_{11}, a_{22}$. Therefore 7 cannot divide four $a_{i}$ 's. This is a contradiction.

Let $k=19$. Now the primes $19,17,13,11,7,5$ divide $1,2,2,2,3,4$ distinct $a_{i}$ 's, respectively. Moreover these $a_{i}$ 's are divisible by only one of the primes given above. Let 17 divide $a_{0}, a_{17}$. If 5 divides $a_{1}, a_{6}, a_{11}, a_{16}$, we observe that 7 cannot divide three $a_{i}$ 's, a contradiction. Thus 5 divides $a_{3}$, $a_{8}, a_{13}, a_{18}$. Then 7 divides $a_{2}, a_{9}, a_{16} ; 13$ divides $a_{1}, a_{14} ; 11$ divides $a_{4}, a_{15}$. Thus one of the elements $a_{5}, a_{6}, a_{7}, a_{10}, a_{11}, a_{12}$ is divisible by 19 and the others are composed only of 2 and 3 . Further we observe that

$$
\left(\frac{a_{5}}{7}\right)=\left(\frac{a_{7}}{7}\right)=\left(\frac{a_{12}}{7}\right)=-\left(\frac{d}{7}\right), \quad\left(\frac{a_{6}}{7}\right)=\left(\frac{a_{10}}{7}\right)=\left(\frac{a_{11}}{7}\right)=\left(\frac{d}{7}\right) .
$$

Let 19 divide $a_{5}$. Then either $a_{7}, a_{12} \in\{3,6\}$ or $a_{6}, a_{10}, a_{11} \in\{3,6\}$, a contradiction. The possibilities of 19 dividing $a_{6}, a_{7}, a_{10}, a_{11}, a_{12}$ are excluded similarly. Hence 17 divides $a_{1}, a_{18}$. If 5 divides $a_{2}, a_{7}, a_{12}, a_{17}$, then 7 cannot divide three $a_{i}$ 's, a contradiction. Therefore 5 divides $a_{0}, a_{5}, a_{10}, a_{15}$. This is excluded as in the case 17 dividing $a_{0}, a_{17}$ and 5 dividing $a_{3}, a_{8}, a_{13}, a_{18}$.

Let $k=17$. The proof depends again as in the cases $k=23$ on that there are exactly 8 distinct $a_{i}$ 's composed only of 2,3 and 5 . We observe that 5 divides $a_{0}, a_{5}, a_{10}, a_{15}$ or $a_{1}, a_{6}, a_{11}, a_{16}$. In the former possibility, 7 divides $a_{2}, a_{9}, a_{16}$ and 13 divides $a_{1}, a_{14}$, which is not possible since 11 cannot divide two $a_{i}$ 's. The latter possibility is excluded similarly.

Let $k=11,13$. There are exactly four distinct $a_{i}$ 's composed only of 2 and 3 . First we consider the case $k=13$. Then the primes $13,11,7,5$ divide exactly $1,2,2,3$ distinct $a_{i}$ 's respectively. Thus 11 divides $a_{0}, a_{11}$ or $a_{1}, a_{12}$. Let 11 divide $a_{0}, a_{11}$. Then 5 divides $a_{2}, a_{7}, a_{12}$ and 7 divides $a_{1}, a_{8}$ or $a_{3}$, $a_{10}$. Let 7 divide $a_{1}, a_{8}$. Then one of $a_{3}, a_{4}, a_{5}, a_{6}, a_{9}, a_{10}$ is divisible by 13 and others are composed of 2 and 3. Considering Legendre symbols modulo 7 and using $|R|=12$, we see that $a_{4}, a_{6} \in\{1,2\}$. Further, if 13 divides $a_{3}$, then $a_{5}, a_{9}, a_{10} \in\{3,6\}$, a contradiction. Similarly we see that 13 cannot divide any of $a_{5}, a_{9}, a_{10}$. The other possibility of 7 dividing $a_{3}, a_{10}$ leads to a similar contradiction. The case of 11 dividing $a_{1}, a_{12}$ is excluded similarly.

Let $k=11$. Then 5 divides $a_{0}, a_{5}, a_{10}$. Further 7 divides $a_{1}, a_{8}$ or $a_{2}, a_{9}$. Let 7 divide $a_{1}, a_{8}$. Then 11 divides one of $a_{2}, a_{3}, a_{4}, a_{6}, a_{7}, a_{9}$ and the remaining ones are divisible by 2 and 3 only. Let 11 divide $a_{6}$. Then we use Legendre symbols modulo 7 as in the case $k=19$ to derive that $a_{4}, a_{7} \in\{3,6\}$ and $a_{2}, a_{3}, a_{9} \in\{1,2\}$. Thus $\left(\frac{a_{3}}{3}\right)=\left(\frac{a_{9}}{3}\right)=-\left(\frac{d}{3}\right)$. Then $a_{3}=a_{9}=1$ or 
$a_{3}=a_{9}=2$. Let $a_{3}=a_{9}=1$. Then $a_{2}=2$, implying that $n$ is even and so $d$ is odd. Thus $n+3 d$ and $n+9 d$ are odd squares, hence congruent to 1 modulo 8. This implies that $4 \mid 6 d$, a contradiction. Therefore $a_{3}=a_{9}=2$. Further $\left(\frac{a_{3}}{11}\right)=-\left(\frac{d}{11}\right)$ and $\left(\frac{a_{9}}{11}\right)=\left(\frac{d}{11}\right)$ since 11 divides $a_{6}$. This is not possible. Let 11 divide $a_{3}$. Then $a_{2}, a_{9} \in\{3,6\}$ or $a_{4}, a_{6}, a_{7} \in\{3,6\}$ by using Legendre symbols modulo 7 . This is not possible. The possibilities of 11 dividing $a_{4}$, $a_{7}, a_{9}$ are excluded similarly to $11 \mid a_{3}$. If 11 divides $a_{2}$, then $a_{3}, a_{9} \in\{3,6\}$ and $a_{4}, a_{6}, a_{7} \in\{1,2\}$. Hence $a_{4}=a_{6}=1, a_{7}=2$ since either $a_{3}$ or $a_{9}$ is 6 . Now we observe that $n+4 d$ and $n+6 d$ are odd squares. Therefore 8 divides $2 d$, which is not possible. Hence 7 does not divide $a_{1} a_{8}$. Similarly we conclude that 7 does not divide $a_{2} a_{9}$.

The next result is due to Pocklington [6].

LEMMA 7. The equation

$$
r^{4}+s^{4}+10 r^{2} s^{2}=z^{2}
$$

does not have any solution in positive integers $r, s, z$ with $\operatorname{gcd}(r, s)=1$ and $r \not \equiv s(\bmod 2)$.

Next we prove Theorem 4 apart from two exceptional cases.

Lemma 8. Equation (1) with $\operatorname{gcd}(n, d)=1, k=5$ and $P(b)<k$ implies that either

$$
n=x_{0}^{2}, \quad n+d=3 x_{1}^{2}, \quad n+2 d=2 x_{2}^{2}, \quad n+3 d=x_{3}^{2}, \quad n+4 d=2 x_{4}^{2}
$$

or

$$
n=2 x_{0}^{2}, \quad n+d=x_{1}^{2}, \quad n+2 d=2 x_{2}^{2}, \quad n+3 d=3 x_{3}^{2}, \quad n+4 d=x_{4}^{2} .
$$

Proof. Assume (1) with $\operatorname{gcd}(n, d)=1, k=5$ and $P(b)<k$. Let $d$ be even. Then $a_{i}=1$ or 3 . If $a_{0}=3$, then $a_{1}=a_{2}=1$, which is not possible since $1=\left(\frac{a_{1}}{3}\right)=\left(\frac{d}{3}\right)$ and $1=\left(\frac{a_{2}}{3}\right)=\left(\frac{2 d}{3}\right)=-\left(\frac{d}{3}\right)$. Thus $a_{0} \neq 3$. Similarly we observe that none of the $a_{i}$ 's is equal to 3 . This contradicts Lemma 4 . Thus $d$ is odd. Let 3 divide $d$. Then $a_{i}=1$ or 2 . This is not possible since there are at least two odd terms. Thus $d \equiv \pm 1(\bmod 6)$. We assume that $d \equiv-1(\bmod 6)$ and we show that $(11)$ holds. Since $(12)$ is the mirror image of (11), it can be shown similarly that $d \equiv 1(\bmod 6)$ implies $(12)$. Thus we restrict ourselves to the case $d \equiv-1(\bmod 6)$.

Now we observe that none of the $n+i d$ with $0 \leq i \leq 4$ is congruent to $5(\bmod 6)$. Therefore $n \equiv 4(\bmod 6)$ since $d \equiv-1(\bmod 6)$. We exclude all the possibilities other than (11) and

$$
\begin{array}{lllll}
a_{0}=1, & a_{1}=3, & a_{2}=2, & a_{3}=1, & a_{4}=1, \\
a_{0}=1, & a_{1}=3, & a_{2}=2, & a_{3}=1, & a_{4}=3, \\
a_{0}=1, & a_{1}=3, & a_{2}=2, & a_{3}=1, & a_{4}=6 .
\end{array}
$$


The last possibility is excluded by Lemma 4 . Next we consider (13). We observe that $x_{0}, x_{4}$ are even, $x_{2}$ is odd and

$$
x_{2}^{2}=\left(\frac{x_{0}}{2}\right)^{2}+\left(\frac{x_{4}}{2}\right)^{2} .
$$

Let $4 \mid x_{0}$. Then $n+4 d \equiv 4(\bmod 8)$ and $n+d \equiv 3(\bmod 8)$, implying that $d \equiv 3(\bmod 8)$. Then $2 x_{2}^{2}=n+2 d \equiv 6(\bmod 8)$, which is not possible. Thus $x_{0} / 2$ is odd and $x_{4} / 2$ is even. Then

$$
\frac{x_{0}}{2}=r^{2}-s^{2}, \quad \frac{x_{4}}{2}=2 r s, \quad x_{2}=r^{2}+s^{2}
$$

where $r>s$ are positive integers such that $\operatorname{gcd}(r, s)=1$ and $r \not \equiv s(\bmod 2)$. Now we use the relation $2 x_{3}^{2}=2 x_{2}^{2}+x_{4}^{2}$ to conclude that $r^{4}+s^{4}+10 r^{2} s^{2}=x_{3}^{2}$, which is not possible by Lemma 7 . Finally, assume (14). Then $d=x_{4}^{2}-x_{1}^{2}$ $=3 x_{1}^{2}-x_{0}^{2}$, implying that $4 x_{1}^{2}=x_{0}^{2}+x_{4}^{2}$. Also $4 x_{2}^{2}=x_{0}^{2}+3 x_{4}^{2}$. This implies (10) again as above, which is not possible by Lemma 7 .

The possibilities (11) and (12) are ruled out by using the following result.

LEMma 9. If $x$ and $y$ are rational numbers satisfying

$$
y^{2}=x^{3}-3504 x-76160
$$

then

$$
(x, y) \in\{(-40,0),(-28,0),(68,0)\} .
$$

Proof. Using SIMATH, we find that the rank of Mordell group of the elliptic curve (15) is 0 and the torsion points are given by (16).

For the next result we introduce the following polynomials:

$$
\begin{aligned}
& f_{1}(X)=X^{6}+20 X^{5}+158 X^{4}+684 X^{3}+1755 X^{2}+2700 X+2250, \\
& f_{2}(X)=X^{6}+10 X^{5}+33 X^{4}-24 X^{3}-430 X^{2}-1200 X-1000, \\
& f_{3}(X)=f_{1}(5 X) \text { and } f_{4}(X)=f_{2}(5 X) .
\end{aligned}
$$

We apply the method of Runge to obtain the following result:

Lemma 10. Let $1 \leq i \leq 4$ and $X$ be a positive integer. If $f_{i}(X)$ is a square of a positive integer, then $X \leq 85$.

Proof. We give a proof for $i=1$. The proofs for the other cases are similar. First we consider $f_{1}(X)=Y_{1}^{2}$ where $Y_{1}$ is a positive integer. We observe that

$$
\left(X^{3}+10 X^{2}+29 X+52\right)^{2}>Y_{1}^{2} .
$$

Further

$$
Y_{1}^{2}-\left(X^{3}+10 X^{2}+29 X+51\right)^{2}=2 X^{3}-106 X^{2}-258 X-351>0
$$

for $X>55$. Thus 


$$
X^{3}+10 X^{2}+29 X+51<Y_{1}<X^{3}+10 X^{2}+29 X+52
$$

for $X>55$. This is not possible since $Y_{1}$ is an integer. Hence $X \leq 55$.

3. Proof of Theorem 4. Assume (1) with $\operatorname{gcd}(n, d)=1, k=5$ and $P(b)<k$. Then either (11) or (12) holds by Lemma 8. Assume (11). Then $x_{4}^{2}+x_{2}^{2}=x_{3}^{2}$ where $x_{2}, x_{3}$ are odd and $x_{4}$ is even. Then $x_{4}=2 r s, x_{2}=r^{2}-s^{2}$ and $x_{3}=r^{2}+s^{2}$ where $r>s$ are positive integers such that $\operatorname{gcd}(r, s)=1$ and $r \not \equiv s(\bmod 2)$. Now $d=x_{4}^{2}-x_{2}^{2}$ gives $d=6 r^{2} s^{2}-r^{4}-s^{4}$ and $3 d=x_{3}^{2}-x_{0}^{2}$ gives $n=x_{0}^{2}=4\left(r^{4}+s^{4}-4 r^{2} s^{2}\right)$. Using $n+d=3 x_{1}^{2}$ we get $3 r^{4}+3 s^{4}-10 r^{2} s^{2}=3 x_{1}^{2}$. Now we obtain an elliptic equation from this relation. Putting $X_{1}=r / s$, $Z_{1}=x_{1} / s^{2}$ we get

$$
Z_{1}^{2}=X_{1}^{4}-\frac{10}{3} X_{1}^{2}+1
$$

We derive (17) with $Z_{1}=x_{3} / s^{2}$ from (12) in a similar way. Thus (17) is always valid. Now we multiply both sides of (17) by $4 X_{1}^{2}$ to get

$$
v^{2}=4 u^{3}-\frac{40}{3} u^{2}+4 u
$$

where $u=X_{1}^{2}, v=2 Z_{1} X_{1}$. By putting $u=(x+40) / 36$ and $v=y / 108$ we get

$$
y^{2}=x^{3}-3504 x-76160 .
$$

By Lemma 9 we get $x=-40,-28,68$, which gives $u=0,1 / 3,3$. Hence $X_{1}^{2}=0,1 / 3,3$, a contradiction as $X_{1}>0$ is rational.

4. Proofs of Theorems 1 and 2. Let $d$ be a power of 2 . We note that $p$ always denotes an odd prime in [7] and in particular in [7, Theorems 2,3]. By [7, Theorem 2], we may assume that $P(b)=k$ and then the assertion of the theorems follows from [7, Theorem 3]. Thus we may suppose that $d=p^{\alpha}$ where $\alpha>0$ is an integer and $p>2$ is a prime. Then $\operatorname{gcd}(n, d)=p^{\beta}$ with $0 \leq \beta<\alpha$ since $d \nmid n$. By dividing both the sides of (1) by $p^{\beta k}$, we may assume that $\operatorname{gcd}(n, d)=1$. This is clear if $\beta k$ is even. If $\beta k$ is odd, then we see that $p$ divides $b$ and the assumption $\operatorname{gcd}(n, d)=1$ is again clear. By Lemma 5 and Theorem 4, we may also suppose that $k \geq 6$. Further we derive from [7, Corollary 1] that $d>104$. Thus $d \geq k-1$ since $k \leq 29$. Let $|R| \leq k-2$. Suppose that there exist $\mu_{0}>\mu_{1}>\mu_{2}$ such that $a_{\mu_{0}}=a_{\mu_{1}}=a_{\mu_{2}}$. Then

$$
(\mu-\nu) d=a_{\nu}\left(x_{\mu}-x_{\nu}\right)\left(x_{\mu}+x_{\nu}\right)
$$

whenever

$$
(\mu, \nu) \in\left\{\left(\mu_{0}, \mu_{1}\right),\left(\mu_{0}, \mu_{2}\right),\left(\mu_{1}, \mu_{2}\right)\right\} .
$$

In fact the above relation is valid with some $(\mu, \nu)$ satisfying (18) such that $d \mid\left(x_{\mu}-x_{\nu}\right)$. Therefore $k-1 \geq \mu-\nu \geq x_{\mu}+x_{\nu}>d$, which is not possible. Now the assumption (7.5) of [7, Lemma 10] is satisfied since $|R| \leq k-2$ and we 
apply [7, Lemma 10] with $h_{1}=1, h_{2}=p^{\alpha}, c=\varepsilon_{1}=\varepsilon_{2}=\chi_{1}=1$. Therefore $d<4(k-1)$. Consequently, $k=29$ and $d=107,109$. Then $n<28(k-1)^{2}$ by (7.8) of [7, Lemma 10]. Now we apply the algorithm of [7, Section 9] to show that (1) does not hold. The details of the application of this algorithm in some particular cases are explained in [7, Lemma 15]. Finally we remark that the arguments from [7] applied above are valid under the assumption $P(b) \leq k$ in place of $P(b)<k$. Hence $|R| \geq k-1$.

4.1. Proof of Theorem 1. Assume that $P(b)<k$. As mentioned above, we may suppose that $k \geq 6$. Let $|R|=k$. Consider $k=6$. Then 5 divides $a_{0}, a_{5}$ and $a_{1}, a_{2}, a_{3}, a_{4}$ is a permutation of $1,2,3,6$. Now the assertion follows from Lemma 4 . The case $k=8$ is excluded similarly. Further $k \neq 7,9$, otherwise there are at least five distinct $a_{i}$ 's composed only of 2 and 3 . Thus we may assume that $|R|=k-1$. The case $k=9$ is excluded as in $[7$, Lemma $7, k=9]$.

Let $k=8$. The cases of 7 dividing $a_{0}, a_{7}$ and 5 dividing $a_{0}, a_{5}$ or $a_{1}, a_{6}$ or $a_{2}, a_{7}$ are excluded as in [7, Lemma $7, k=8$ ]. Thus it remains to consider the following cases.

(a) 7 divides only one $a_{i}$ and 5 divides two distinct $a_{i}$ 's not divisible by 7 .

(b) 7 divides $a_{0}, a_{7}$ and 5 divides only one $a_{i}$ other than $a_{0}, a_{7}$.

(a) Let 5 divide $a_{0}, a_{5}$. Suppose $7 \mid a_{6}$. Then $a_{1}, a_{2}, a_{3}, a_{4}$ are composed of 2 and 3. This is not possible by Lemma 5 with $n$ replaced by $n+d$. Thus $7 \nmid a_{6}$. Similarly $7 \nmid a_{7}$. Let 7 divide $a_{1}$. Then $a_{2}, a_{3}, a_{4}, a_{6}, a_{7} \in\{1,2,3,6\}$. Now $\left(\frac{a_{2}}{7}\right)=\left(\frac{a_{3}}{7}\right)=\left(\frac{d}{7}\right),\left(\frac{a_{4}}{7}\right)=\left(\frac{a_{6}}{7}\right)=\left(\frac{a_{7}}{7}\right)=-\left(\frac{d}{7}\right)$. So either $a_{2}, a_{3} \in$ $\{3,6\}$ or $a_{4}, a_{6}, a_{7} \in\{3,6\}$. This is not possible. Let 7 divide $a_{2}$. Then $a_{1}, a_{3}, a_{4}, a_{6}, a_{7} \in\{1,2,3,6\}$. Using Legendre symbols modulo 7 we observe that $a_{1}, a_{7} \in\{3,6\}$ and $a_{3}, a_{4}, a_{6} \in\{1,2\}$. Further we see that $\left(\frac{a_{1}}{5}\right)=\left(\frac{a_{4}}{5}\right)=$ $\left(\frac{a_{6}}{5}\right)=\left(\frac{d}{5}\right),\left(\frac{a_{3}}{5}\right)=\left(\frac{a_{7}}{5}\right)=-\left(\frac{d}{5}\right)$. Therefore $a_{1}, a_{4}, a_{6} \in\{1,6\}, a_{3}, a_{7} \in$ $\{2,3\}$ or $a_{1}, a_{4}, a_{6} \in\{2,3\}, a_{3}, a_{7} \in\{1,6\}$. Thus we conclude $a_{1}=6, a_{3}=2$, $a_{4}=1, a_{6}=1, a_{7}=3$ or $a_{1}=3, a_{3}=1, a_{4}=2, a_{6}=2, a_{7}=6$. Since $d$ is odd, we observe that the relations $a_{4}=a_{6}=1$ and $a_{6}=2, a_{7}=6$ do not hold. Therefore both the possibilities are ruled out. We exclude similarly by using Lemma 5 and congruences as above the cases when 5 divides $a_{0}, a_{5}$ and 7 divides $a_{4} ; 5$ divides $a_{1}, a_{6} ; 5$ divides $a_{2}, a_{7}$ and 7 divides $a_{0} a_{1} a_{3} a_{5} a_{6}$. It remains to consider only the cases where 5 divides $a_{0}, a_{5}$ and 7 divides $a_{3}$; 5 divides $a_{2}, a_{7}$ and 7 divides $a_{4}$. Let 5 divide $a_{0}, a_{5}$ and 7 divide $a_{3}$. Then we derive as above that either $a_{1}=1, a_{2}=2, a_{4}=6, a_{6}=1, a_{7}=3$ or $a_{1}=2, a_{2}=1, a_{4}=3, a_{6}=2, a_{7}=6$. The latter possibility is excluded since $a_{1}=a_{6}=2$ is not possible by $d$ odd. The former one gives $x_{6}^{2}-x_{1}^{2}=5 d$ by (2). Thus

$$
d=2 x_{1}+5 \quad \text { or } \quad d=\frac{2 x_{1}+1}{5} .
$$


Also

$$
(n+2 d)(n+4 d)(n+7 d)=\left(6 x_{2} x_{4} x_{7}\right)^{2}=: Y_{1}^{2},
$$

hence we get the following two equations:

$$
\begin{aligned}
& x_{1}^{6}+20 x_{1}^{5}+158 x_{1}^{4}+684 x_{1}^{3}+1755 x_{1}^{2}+2700 x_{1}+2250=Y_{1}^{2}, \\
& x_{1}^{6}+4 x_{1}^{5}+\frac{158}{25} x_{1}^{4}+\frac{684}{125} x_{1}^{3}+\frac{351}{125} x_{1}^{2}+\frac{108}{125} x_{1}+\frac{18}{125}=Y_{1}^{2} .
\end{aligned}
$$

The left hand side of the first one is $f_{1}\left(x_{1}\right)$ of Lemma 10 , and that of the second one becomes $f_{1}\left(5 x_{1}\right)$ on multiplication by $125^{2}$. Thus in both the cases Lemma 10 implies $x_{1} \leq 85$. Now we observe that $\left(\frac{2}{p}\right)=\left(\frac{3}{p}\right)=\left(\frac{5}{p}\right)=$ $\left(\frac{7}{p}\right)=\left(\frac{n}{p}\right)=1$ since $a_{5}=5, a_{3}=7$. Therefore $p \geq 311$, implying $x_{1} \geq 150$, a contradiction. The case of 5 dividing $a_{2}, a_{7}$ and $7 \mid a_{4}$ is ruled out similarly using Lemma 10 with $i=2$. We shall apply Lemma 10 again in the proof of Theorem 2. It is remarkable that 1 is the repeated term among $a_{i}$ 's in all these instances and this is crucial for applying Lemma 10.

(b) By looking modulo 7, we find that either $5 \mid a_{2}$ or $5 \mid a_{5}$. These possibilities are excluded by applying Lemma 5 to products $(n+3 d)(n+4 d)$ $\times(n+5 d)(n+6 d)$ and $(n+d)(n+2 d)(n+3 d)(n+4 d)$, respectively.

Let $k=7$. Then $|R|=6$ and 5 divides two distinct $a_{i}$ 's. Thus 5 divides $a_{0}, a_{5}$ or $a_{1}, a_{6}$. These cases are excluded by Lemma 5 .

Let $k=6$. Then $|R|=5$. The possibility of 5 dividing $a_{0}, a_{5}$ is excluded by Lemma 5 . Thus 5 divides exactly one $a_{i}$. Now we may assume that 5 divides $a_{2}$ or $a_{3}$ by Lemma 5 . Let $5 \mid a_{2}$. By using Legendre symbols modulo 5 , we observe that either $a_{0}, a_{4}, a_{5} \in\{1,6\}, a_{1}, a_{3} \in\{2,3\}$ or $a_{0}, a_{4}, a_{5} \in\{2,3\}$, $a_{1}, a_{3} \in\{1,6\}$. Assume the first possibility. Then $a_{5}=6$, implying neither $a_{1}$ nor $a_{3}$ is equal to 3 . This is not possible. Similarly we see from the second possibility that $a_{1}=a_{3}=1$, contradicting $|R|=5$. The case of 5 dividing $a_{3}$ is excluded similarly.

4.2. Proof of Theorem 2. Assume that $P(b)=k$. By Lemma 6, it remains to consider only the case $k=7$. Then $|R| \geq 6$. We observe that at least one $a_{i}$ is divisible by 5 . We divide the proof into the following two parts:

(a) 5 divides exactly two elements.

(b) 5 divides only one element.

(a) Let 5 divide $a_{0}, a_{5}$. Then we derive from Lemma 5 that 7 does not divide $a_{0}, a_{5}, a_{6}$. By applying Legendre symbols modulo 7 and 5 as in the proof of the case $k=8,5\left|a_{0}, a_{5}, 7\right| a_{2}$ of Theorem 1 , the possibilities of 7 dividing $a_{1}, a_{2}, a_{4}$ are excluded and 7 dividing $a_{3}$ gives

$$
a_{1}=a_{6}=1, \quad a_{2}=2, \quad a_{4}=6 .
$$


Further we conclude as above that $5 \nmid a_{1} a_{6}$ unless 7 divides $a_{3}$ and

$$
a_{0}=a_{5}=1, \quad a_{2}=6, \quad a_{4}=2 .
$$

(b) We observe that $|R|=6$ such that 7 and 5 do not divide the same $a_{i}$. Let 7 divide $a_{0}$. Then we derive from Lemma 5 that 5 cannot divide $a_{1}, a_{2}, a_{5}, a_{6}$. The remaining cases $5 \mid a_{3}$ and $5 \mid a_{4}$ are excluded by considering Legendre symbols modulo 7 and 5 . Thus 7 does not divide $a_{0}$. Let 7 divide $a_{1}$. By considering Legendre symbols modulo 7 and 5 we get

$$
a_{0}=2, \quad a_{2}=6, \quad a_{4}=1, \quad a_{5}=3, \quad a_{6}=2 .
$$

By (2), we have

$$
n(n+2 d)(n+4 d)(n+6 d)=6\left(2 x_{0} x_{2} x_{4} x_{6}\right)^{2} .
$$

We observe that $n$ and $x_{4}$ are even. We divide both sides by $2^{4}$ to obtain

$$
\frac{n}{2}\left(\frac{n}{2}+d\right)\left(\frac{n}{2}+2 d\right)\left(\frac{n}{2}+3 d\right)=6\left(x_{0} x_{2} \frac{x_{4}}{2} x_{6}\right)^{2}
$$

and $\operatorname{gcd}(n / 2, d)=1$. By Lemma 5 we get $n / 2=75$ and $d=23$, implying $x_{0}^{2}=75$, which is not possible. Similarly we show that none of the other $a_{i}$ is divisible by 7 unless

$$
a_{0}=a_{5}=1, \quad a_{1}=2, \quad a_{3}=6, \quad a_{6}=3
$$

in the case $7\left|a_{2}, 5\right| a_{4}$ and

$$
a_{0}=3, \quad a_{1}=a_{6}=1, \quad a_{3}=6, \quad a_{5}=2
$$

in the case $7\left|a_{4}, 5\right| a_{2}$. Further we observe that $a_{2}=7, a_{4}=5$ and $a_{2}=5$, $a_{4}=7$ in (21) and (22), respectively.

It remains to show that the relations (19)-(22) are not valid. First we consider (19). From (2) we get

$$
n+d=x_{1}^{2}, \quad n+2 d=2 x_{2}^{2}, \quad n+4 d=6 x_{4}^{2}, \quad n+6 d=x_{6}^{2} .
$$

So $5 d=x_{6}^{2}-x_{1}^{2}$, implying either $x_{6}-x_{1}=1, x_{6}+x_{1}=5 d$ or $x_{6}-x_{1}=5$, $x_{6}+x_{1}=d$. In the first case $5 d=2 x_{1}+1$, which gives the following equations:

$$
X^{2}+4=2 Y^{2}, \quad(X+2)^{2}+6=6 Z^{2}
$$

with $X=5 x_{1}+1, Y=5 x_{2}$ and $Z=5 x_{4}$. In the latter case $d=2 x_{1}+5$ and we get the same pair of Pell's equations with $X=x_{1}+1, Y=x_{2}$ and $Z=x_{4}$. This is not possible by Lemma 3 . The case (20) is excluded similarly again by Lemma 3 . Next, we consider (21). By (2), we have

$$
\begin{gathered}
n=x_{0}^{2}, \quad n+d=2 x_{1}^{2}, \quad n+2 d=7 x_{2}^{2}, \quad n+3 d=6 x_{3}^{2}, \\
n+4 d=5 x_{4}^{2}, \quad n+5 d=x_{5}^{2}, \quad n+6 d=3 x_{6}^{2},
\end{gathered}
$$


which implies that

$$
\left(\frac{n}{p}\right)=\left(\frac{2}{p}\right)=\left(\frac{3}{p}\right)=\left(\frac{5}{p}\right)=\left(\frac{7}{p}\right)=1,
$$

where $d=p^{\alpha}$ with positive integer $\alpha$. Further either $5 d=2 x_{0}+1$ or $d=$ $2 x_{0}+5$. We also observe that

$$
(n+d)(n+3 d)(n+6 d)=\left(6 x_{1} x_{3} x_{6}\right)^{2} .
$$

Putting the expressions for $n$ and $d$ in terms of $x_{0}$, we get

$$
f_{1}(X)=Y^{2} \quad \text { with } \quad X=x_{0}, Y=6 x_{1} x_{3} x_{6}
$$

if $d=2 x_{0}+5$, or

$$
f_{1}(X)=Y^{2} \quad \text { with } \quad X=5 x_{0}, Y=750 x_{1} x_{3} x_{6}
$$

if $5 d=2 x_{0}+1$. We observe that (22) is the mirror image of (21), and therefore, it implies similarly

$$
f_{2}(X)=Y^{2} \quad \text { with } \quad X=x_{1}, Y=6 x_{0} x_{3} x_{5}
$$

and

$$
f_{2}(X)=Y^{2} \quad \text { with } \quad X=5 x_{1}, Y=750 x_{0} x_{3} x_{5}
$$

We recall that (24) and (25), together with (23), are excluded by Lemma 10 with $i=1$ in the proof of the case (a) of $k=8$ in Theorem 1. Further (26) and (27) are excluded similarly by applying Lemma 10 with $i=2$.

\section{References}

[1] A. Baker and H. Davenport, The equations $3 x^{2}-2=y^{2}$ and $8 x^{2}-7=z^{2}$, Quart. J. Math. Oxford Ser. (2) 20 (1969), 129-137.

[2] A. Baker and G. Wüstholz, Logarithmic forms and group varieties, J. Reine Angew. Math. 442 (1993), 19-62.

[3] L. E. Dickson, History of the Theory of Numbers, Vol. II, Chelsea, 1952.

[4] L. J. Mordell, Diophantine Equations, Academic Press, 1969.

[5] R. Obláth, Über das Produkt fünf aufeinander folgender zahlen in einer arithmetischen Reihe, Publ. Math. Debrecen 1 (1950), 222-226.

[6] H. C. Pocklington, Some Diophantine impossibilities, Proc. Cambridge Philos. Soc. 17 (1912), 108-121.

[7] N. Saradha and T. N. Shorey, Almost squares in arithmetic progression, Compositio Math., to appear.

[8] T. N. Shorey, Exponential diophantine equations involving products of consecutive integers and related equations, in: Number Theory, R. P. Bambah, V. C. Dumir and R. J. Hans-Gill (eds.), Hindustan Book Agency, 1999, 463-495.

[9] - , Powers in arithmetic progression, in: A Panorama in Number Theory or The View from Baker's Garden, G. Wüstholz (ed.), Cambridge Univ. Press, 2002, 325336.

[10] T. N. Shorey and R. Tijdeman, Perfect powers in products of terms in an arithmetical progression, Compositio Math. 75 (1990), 307-344. 
[11] R. Tijdeman, Diophantine equations and diophantine approximations, in: Number Theory and Applications (Banff, AB, 1988), R. A. Mollin (ed.), NATO Adv. Sci. Inst. Ser. C Math. Phys. Sci. 265, Kluwer, 1989, 215-243.

Harish-Chandra Research Institute

Allahabad 211019, India

E-mail: anirban@mri.ernet.in
School of Mathematics Tata Institute of Fundamental Research Homi Bhabha Road Mumbai 400005, India E-mail: shorey@math.tifr.res.in

Received on 27.11.2001

and in revised form on 5.3.2003 